\title{
Identification of a new mouse sperm acrosome-associated protein
}

\author{
Joanna Korfanty, Agnieszka Toma, Aleksandra Wojtas, Aleksandra Rusin, Natalia Vydra and \\ Wieslawa Widlak
}

Center for Translational Research and Molecular Biology of Cancer, Maria Skłodowska-Curie Memorial Cancer Center and Institute of Oncology, Gliwice Branch, Wybrzeże Armii Krajowej 15, 44-101 Gliwice, Poland

Correspondence should be addressed to W Widlak; Email: wwidlak@io.gliwice.pl

\begin{abstract}
The binding of capacitated spermatozoa to the egg's extracellular coat and induction of acrosome reaction are necessary for successful fertilization in mammals. Biogenesis of acrosome is complicated, and not all proteins involved in this process are known. In this study, we have cloned a novel mouse gene, Spaca7, that is expressed exclusively in the testes. During the postnatal development, transcripts of the gene could be detected at a very low level in 18-day-old mouse testes and at a higher level in 21-day-old mouse testes and later, which corresponds to an expansion of round spermatids. In the stably transfected PT67 cells, SPACA7 fused with EGFP was predominantly localized in the Golgi apparatus. In transgenic mouse testes, the fusion protein was found in acrosome (starting from the first stages of acrosome formation in late pachytene spermatocytes and finally in spermatozoa isolated from caput and cauda of epididymis). Confocal microscopy studies revealed an intra-acrosomal not membrane-bound localization of SPACA7/EGFP, which suggests that the protein can be released during acrosome reaction and involved in fertilization. Acrosomal localization of endogenous SPACA7 protein was also found in human spermatozoa.
\end{abstract}

Reproduction (2012) 143 749-757

\section{Introduction}

Mammalian spermatozoa penetrate the egg's coats using a number of hydrolytic enzymes accumulated in a secretory vesicle termed the acrosome (Primakoff \& Myles 2002). Hence, functional sperm requires properly formed acrosomes, and males carrying mutations that affect their formation are infertile or show subfertility (Yan 2009). The acrosome is a lysosome-like membranebound organelle covering the head of spermatozoon. In mammals, acrosome biogenesis begins in the late pachytene spermatocyte and continues throughout the first half of spermiogenesis. Biogenesis of the acrosome can be divided into three phases: Golgi, cap, and acrosomal (Abou-Haila \& Tulsiani 2000). During the Golgi phase, numerous proacrosomal granules synthesized by the Golgi apparatus fuse with one another to form a single large acrosomal vacuole that is in contact with the nuclear envelope. During the cap phase, new proteins from the Golgi apparatus are still added, and the growing acrosomal vesicle flattens and spreads over the nucleus, covering up to two-thirds of its surface. During the acrosomal phase, proteins condense and are packed in a paracrystalline structure called the acrosomal granule, which condenses and applies itself to the inner acrosomal membrane. In late spermatids, no new proteins are added to the acrosome (Braun et al. 1989). The structure assumes a hemispherical, cap-like shape and maintains this shape thereafter until the acrosomal reaction has been completed.

During spermatogenesis, germ cells exhibit a specific and complex pattern of gene expression. This specificity is manifested by expression of genes coding for testisspecific variants of somatic proteins, or unique genes that code for proteins not synthesized in somatic cells (Lee et al. 2009). It was estimated that more than $50 \%$ of the mouse genome would be expressed during testis development from birth to adulthood, and about $4 \%$ is transcribed only in male germ cells (Schultz et al. 2003). Many of these genes encode proteins involved in acrosome formation, sperm-egg interactions, and fertilization, yet a large fraction of them remains uncharacterized so far.

Besides well-characterized proteins involved in fertilization, enzymes (proteases, glycosidases, esterases, acid phosphatases, aryl sulfatases), antigens, and various ZP-binding proteins (Buffone et al. 2008), the acrosome contains some less-characterized proteins grouped in sperm acrosome associated (SPACA) family proteins. This family has been created based on the cellular 
localization of its members, but the function of some family members is still speculative. In addition to localization in the acrosomal matrix, association with the acrosomal membrane was reported for SPACA1 and SPACA4 (Hao et al. 2002, Shetty et al. 2003) and suggested for SPACA3 (Herrero et al. 2005). The most extensively studied SPACA3 and SPACA4 could be involved in the binding of spermatozoa to the egg plasma membrane (Shetty et al. 2003, Herrero et al. 2005). SPACA3 has been shown to be a lysozyme-like protein and has the conserved substrate-binding sites for the oligosaccharides of $\mathrm{N}$-acetylglucosamine. This could enable its binding to hyaluronan and/or related molecules in the perivitelline space of the egg (Mandal et al. 2003). SPACA4 is a glycosylphosphatidylinositolanchored protein and possesses a functional domain similar to the Ly- 6 and urokinase plasminogen activator receptor superfamily, which suggests that SPACA4 may function as an acrosomal membrane receptor and participate in proteolytic or adhesive events involving the acrosomal membrane (Shetty et al. 2003). Within the SPACA family, only SPACA3 and SPACA5 have strong amino acid homology (within conserved lysozyme-like domains).

Here, we report cloning and the initial characterization of the novel testis-specific gene, Spaca7, that codes for protein localized specifically in acrosome. We paid attention to the Spaca7 (1700094C09Rik) gene after the microarray-based (Affymetrix platform) analysis of global gene expression profiles in mouse spermatogenic cells, which revealed high level of its expression in these cells. Although SPACA7 was included in the SPACA family, the protein has apparently distinct sequence features compared with other members of the family.

\section{Results}

Mouse Spaca7 (RIKEN cDNA 1700094C09) gene is located on chromosome 8 . It has an open reading frame encoding putative, uncharacterized protein C13orf28 homolog precursor (formerly: hypothetical protein LOC78634 precursor). The hypothetical protein consists of 182 amino acids, its calculated molecular weight is 19.930 Da, and is negatively charged (-14.5), assuming that $\mathrm{N}$ and $\mathrm{C}$ termini are not blocked (http://www. encorbio.com/protocols/Prot-MW.htm). The Spaca7 gene is conserved in humans, chimpanzees, and rats. None of the proteins encoded by Spaca7 gene orthologs in these species have any functional annotations. Searching for possible functions of a SPACA7 protein, we analyzed its hypothetical structure using MyHits motif scan (http://myhits.isb-sib.ch/cgi-bin/motif_scan, Sigrist et al. 2010), which allowed the identification of putative anaphylotoxin-like domain motif (ANATO, 17-30aa, $E$ value $=0.00023)$. WoLF PSORT program (http://wolfpsort.seq.cbrc.jp/; Horton et al. 2007) used for protein subcellular localization prediction indicated that the SPACA7 is most likely an extracellular protein.

We analyzed the Spaca7 expression by RT-PCR in mouse liver, testes, and several cell lines (LL2, HECa1, NIH3T3, B16-F10, C26, Renca). The analysis revealed that Spaca7 was expressed only in testis (Fig. 1A). This is in agreement with the data presented in the Gene Expression Atlas (http://www.ebi.ac.uk/gxa/gene/ ENSMUSG00000010435). During postnatal development, a very weak expression of Spaca7 could be detected in the testes of 18-day-old mice, when late pachytene spermatocytes appeared (Bellvé et al. 1977), and its higher expression was observed in 21-day-old animals, when round spermatids developed (Fig. 1B).

To study SPACA7 intracellular localization, we have cloned its CDNA in a frame with the EGFP coding sequence. For expression in somatic cells, the Spaca7/EGFP fusion construct was cloned in pLNCX2 plasmid and introduced into Retropack PT67 cells. In stably transfected cells, the EGFP fluorescence was preferentially concentrated in Golgi apparatus (Fig. 2A). In some cells, strong accumulation of the SPACA7/EGFP was observed and green fluorescence was also visible as spots (vesicles) located peripherally (Fig. 2D). Occasionally, green spots were found outside the cell (Fig. 2G), which suggests that SPACA7/EGFP could be released from the cell.

For expression specifically in spermatogenic cells, the Spaca7/EGFP fusion construct was cloned under control of the rat Hspa2 promoter, and transgenic mice were generated. The Hspa2 gene is expressed at a high level in spermatocytes and spermatids (Krawczyk et al. 1988), and Hspa2 promoter fragment used in this study (1133-bp long) can drive EGFP reporter gene expression mainly into the testis (Vydra et al. 2009). To overcome possible position effects, we introduced insulator

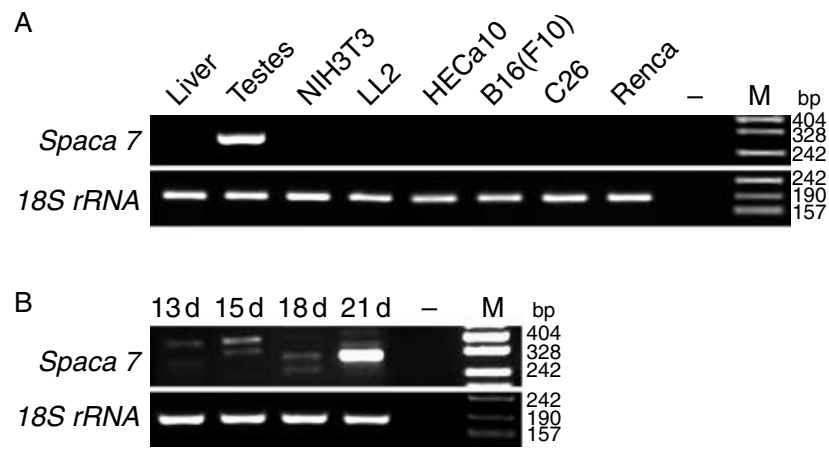

Figure 1 RT-PCR analysis of the Spaca7 expression. (A) Expression in adult mice livers, testes, and in several murine cell lines. (B) Expression in testes during postnatal development (13- to 21-day old). To visualize weak expression in testes of young animals, 36 cycles of PCR were performed (this resulted in an appearance of unspecific bands; compare with Fig. 3). Loading control reactions were performed with primers specific to $18 S$ rRNA transcript. M, marker (sizes of DNA fragments are given in the right side); - , PCR-negative control without template. 

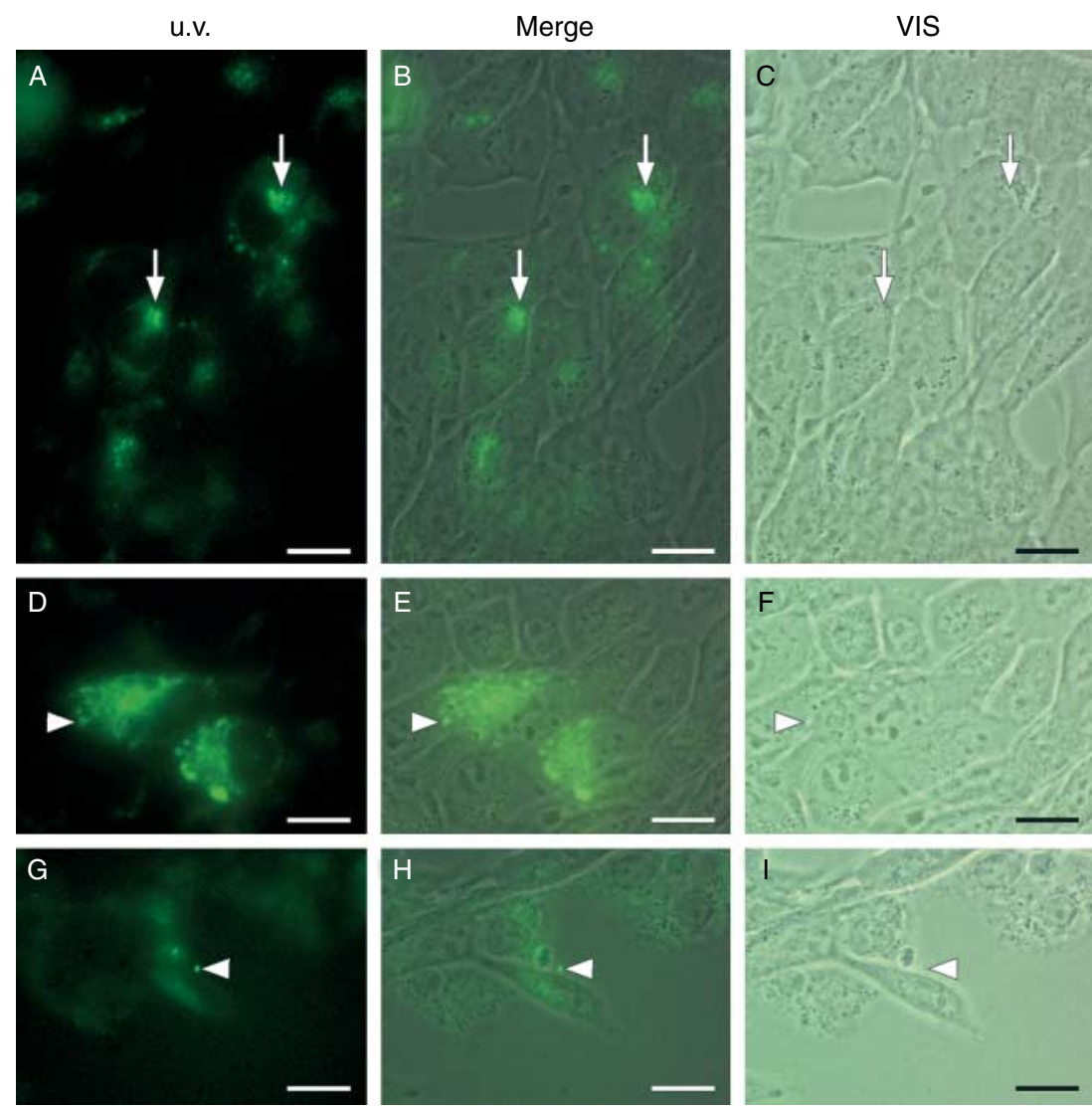

Figure 2 Localization of SPACA7/EGFP fusion protein in living, stably transfected PT67 cells. (A) EGFP fluorescence is concentrated predominantly in Golgi apparatus (arrows). (D) In some cells, EGFP is visible in vesicles at the edge of the cell (arrowhead) or (G) as a green spot outside the cell (arrowhead). (C, F, and I) Bright field images corresponding to $A, D$, and $G$ respectively. ( $B, E$, and $H$ ) Merge of u.v. and VIS images; scale bar $=20 \mu \mathrm{m}$. sequences from the chicken $\beta$-globin locus into the transgene (Recillas-Targa et al. 2002). We established three transgenic lines with the pHspa2-Spaca7/EGFP transgene. In two lines, more than one copy was integrated as a tandem repeat (in this case, only the end copy of the transgene would be susceptible to a positional effect). It was confirmed by PCR analysis with primers complementary to insulator sequences (not shown). In all three transgenic lines, the pattern of the transgene expression was similar, although the level of the expression was slightly different between them. Expression of the pHspa2-Spaca7/EGFP transgene was analyzed in the course of the postnatal development of mouse testes (Fig. 3). The endogenous Spaca7 gene was expressed at a similar level in wild-type and transgenic testes. The transgene expression started slightly earlier than endogenous Spaca7 gene expression: in 15-day-old mouse testes, when Hspa2 promoter was activated in spermatocytes. Additional copies of the Hspa2 promoter did not markedly change the expression of the endogenous Hspa2 gene. Overexpression of the SPACA7/EGFP fusion protein did not influence male fertility and testis morphology was correct.

The fluorescence of the SPACA7/EGFP could be noticed as a weak spot in some spermatocytes (Fig. 4Aa), stronger spots grouped together in early stages of round spermatids (Fig. 4Ab) or as a cap-like shape in later stages of spermatids (Fig. 4Ac), resembling the way of acrosome formation. Finally, EGFP fluorescence originating from fusion protein was found in spermatozoa isolated from caput or cauda of epididymis as a sickle shape located at the tip of the head (Fig. 5A). In transgenic mice line with the highest transgene expression, an additional green spot moving toward the tip of the spermatozoa could be visible (Fig. 5B). In contrast, green fluorescence originating from EGFP protein alone, in $\mathrm{Tg}(\mathrm{Hspa2}-\mathrm{EGFP})$ mice, was uniformly distributed in spermatozoa (Fig. 5C). Confocal microscopy studies revealed that the SPACA7/EGFP fusion protein is uniformly distributed in the acrosome and not limited to the acrosomal membrane (Fig. 5D).

Endogenous SPACA7 localization was further investigated on fixed spermatozoa. Commercially available antibody used in the study was raised against synthetic peptide corresponding to the $\mathrm{N}$-terminal domain of the human SPACA7 protein. Because the antibody did not react with mouse protein (not shown), the localization of endogenous SPACA7 protein was studied on human spermatozoa. Light microscopic immunocytochemistry localized the SPACA7 protein to a cap-shaped domain corresponding to the acrosome of human sperm heads (Fig. 6). 


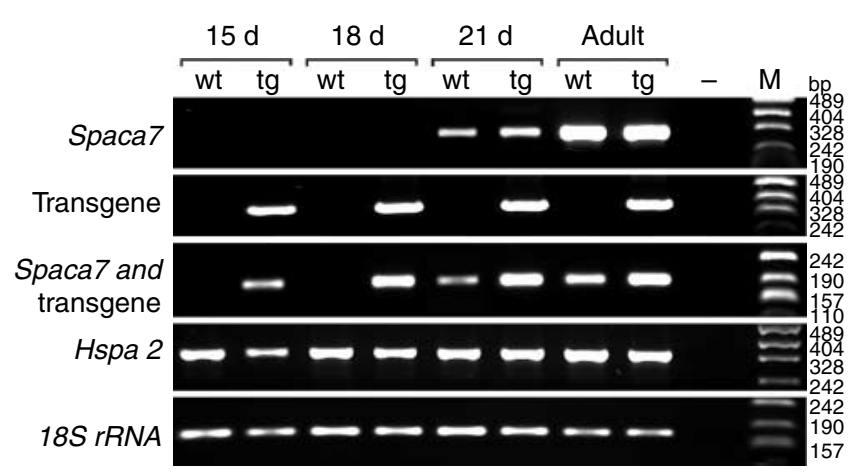

Figure 3 RT-PCR analysis of the $p H$ spa2-Spaca7/EGFP transgene expression in testes during postnatal development of mice. PCR starters were selected to amplify either endogenous Spaca7 or Spaca7/EGFP transgene or both. Loading control reactions were performed with primers specific to $18 S \mathrm{rRNA}$ transcript. $\mathrm{M}$, marker (sizes of DNA fragments are given in the right side); -, PCR-negative control without template; wt, wild-type mice; $\mathrm{tg}$, transgenic mice.

\section{Discussion}

SPACA7 has orthologs in rats, humans, chimpanzees, macaques, and orangutans. However, no putative conserved domains have been detected in these proteins and their function is yet unknown. Here, we depicted the localization of SPACA7 as a fusion protein with EGFP and directly by immunocytochemistry. Observed localization of this protein suggests that SPACA7 is a component of the acrosomal matrix and could be associated with acrosome formation. Thus, for the gene known until now as 1700094C09Rik, a new name, sperm acrosome associated 7, Spaca7, was approved by the International Committee on Standardized Genetic Nomenclature for Mice and the Mouse Genomic Nomenclature Committee.

Observed SPACA7/EGFP distribution in testes of transgenic mice exactly matches the acrosome formation, while ectopic expression of the fusion protein in somatic cells results in localization in Golgi apparatus. In spermatogenic cells, there is a close relationship between the forming acrosomal vesicle and the Golgi apparatus. At the first stages of acrosome biogenesis, the Golgi is actively engaged in the formation of the acrosomal vesicle, producing and delivering the proteins and membranes needed for its enlargement and differentiation. However, at later stages of spermiogenesis, the Golgi apparatus migrates toward the cell pole opposite the nascent acrosome, while the acrosomal vesicle lies underneath the plasma membrane. Eventually, the Golgi apparatus remains in the cytoplasmic lobe and is discarded in the cytoplasmic droplet along with most of the other organelles (Moreno et al. 2000). Finally, our observations on SPACA7/EGFP localization in spermatogenic cells reveal that SPACA7 is strictly an acrosomal protein - it was not observed in Golgi when it separates from the acrosome.

The presence of SPACA7 in the acrosome is somewhat consistent with its putatively extracellular localization predicted on the basis of amino acids sequence (http:// wolfpsort.seq.cbrc.jp/; Horton et al. 2007). Similarly, observation of the SPACA7/EGFP fusion protein in the
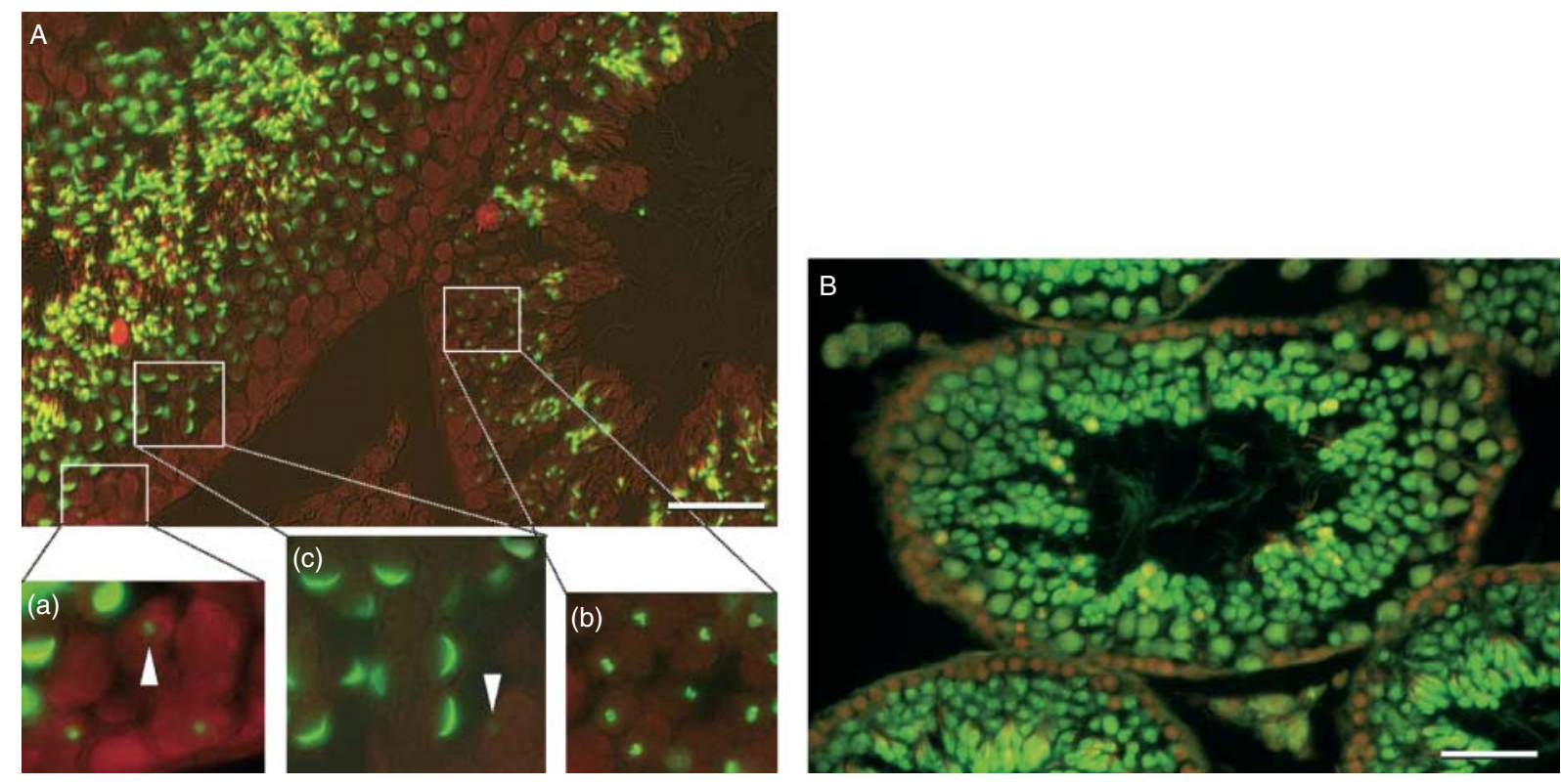

Figure 4 Localization of SPACA7/EGFP fusion protein in transgenic mouse testis. (A) EGFP fluorescence in testis of Tg(Hspa2-Spaca7/EGFP) mice showing distribution of SPACA7/EGFP fusion protein $(a, b$, and $c)$; picture presented in (a) was overexposed to show weak fluorescence in late pachytene spermatocytes (arrowheads in a and c). (B) EGFP fluorescence in testis of $\mathrm{Tg}(\mathrm{Hspa2}$-EGFP) mouse showing a uniform distribution of EGFP alone in cells activating the Hspa2 promoter (spermatocytes and spermatids). Testes were formalin fixed and paraffin embedded. Counterstaining with propidium iodide was performed to better visualize cells outlines. Scale bar $=50 \mu \mathrm{m}$. 

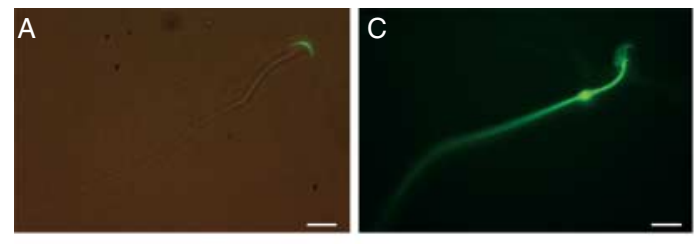

B
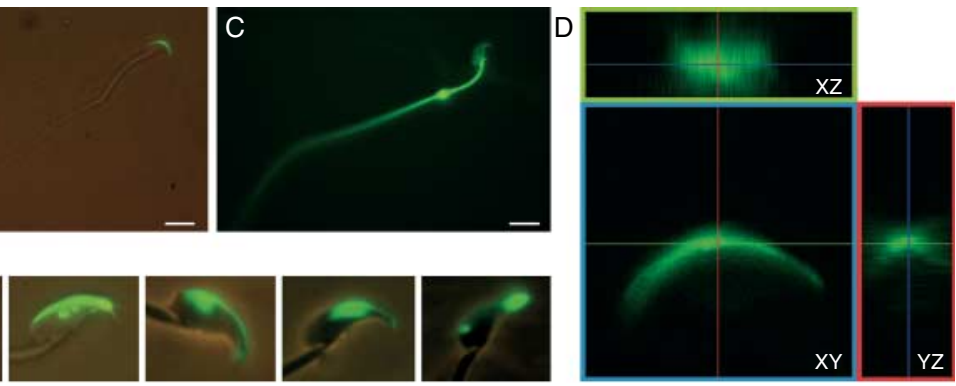

Figure 5 Localization of SPACA7/EGFP fusion protein in mouse spermatozoa extracted from caput of epididymis. (A) EGFP fluorescence in spermatozoon of $\mathrm{Tg}(\mathrm{H}$ spa2-Spaca7/EGFP) transgenic mouse (u.v. + VIS light). (B) Examples of SPACA7/EGFP fusion protein distribution in the head of spermatozoa. (C) Control EGFP fluorescence in spermatozoon of Tg(Hspa2-EGFP) mouse (u.v. light). (D) Orthogonal projection of confocal sections of a representative head of spermatozoon with SPACA7/EGFP protein localized inside the acrosome. The main image shows a section of the acrosome in the XY plane, the $\mathrm{Z}$ position is indicated by the blue lines in the $\mathrm{XZ}$ plane (small top panel), and the $\mathrm{YZ}$ plane (small right-side panel). Scale bar $=10 \mu \mathrm{m}$.

PT67 cells indicated its ability to be exported outside the cell. It is noteworthy that cells overexpressing SPACA7 required more frequent changes of medium to keep them vital, as compared with control cells stably transfected with an empty vector (not shown). This suggested that mature SPACA7 protein secreted from cells might gain proteolytic activity or other activities that lead to cell detachment and premature death in a culture dish (anoikis). The analysis of SPACA7 using MyHits motif scan (Sigrist et al. 2010) indicated the presence of a putative anaphylotoxin-like domain motif in its $\mathrm{N}$-terminal domain that would be responsible for such activities. Anaphylotoxins (C3a, C4a, and $\mathrm{C} 5 \mathrm{a}$ ) are highly hydrophilic protein fragments generated enzymatically in serum during activation of complement molecules C3, C4, and C5. They are able to trigger degranulation (release of substances, e.g. histamine) of endothelial cells, mast cells, or phagocytes and mediate chemotaxis, inflammation, and generation of cytotoxic oxygen radicals (Gennaro et al. 1986, Rosa et al. 1989). If SPACA7 indeed acquires proteolytic or anaphylotoxin activity after release from the cell, it might be involved in the penetration of the zona pellucida, but this hypothetical possibility requires further verification. However, orthologs of SPACA7 in other species probably do not contain anaphylotoxin-like domain motif (according to the MyHits motif scan). Thus, it is not clear whether this domain in mice SPACA7 have any functional importance.

Because we did not find any amino acid sequence similarity between SPACA7 and other members of the SPACA family, we were looking for some other common features beside protein localization. Spaca genes are expressed in a similar way, so we checked the possibility that their transcription could be regulated by a common regulatory protein. We have done in silico promoters analysis, searching for transcription factor binding sites (based on TFSEARCH database; Heinemeyer et al. 1998; Fig. 7). Analysis revealed that proximal promoters of all Spaca genes contain consensus binding sites (with a very high score) for sex determining region of Chr Y (SRY; testis-determining factor). Sry is a member of the SRY-like box (SOX) gene family of DNA-binding proteins. It has been shown to be expressed in adult testes (Zwingman et al. 1994, Modi et al. 2005). Similarly, Sox5 expression,

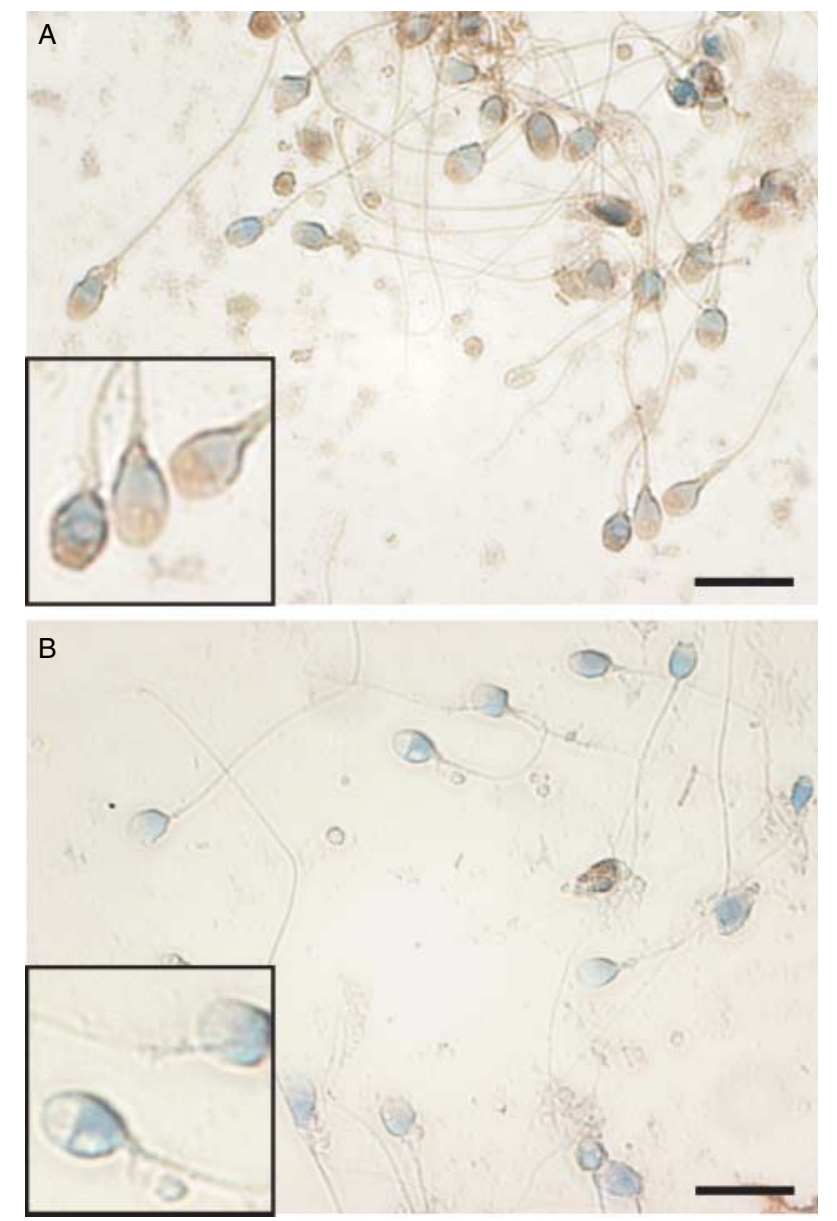

Figure 6 Light microscopic immunocytochemistry for human SPACA7 of ejaculated sperm, counterstained with hematoxylin. Staining (brown) is confined to the acrosome (A). (B) Control staining without specific primary antibody. Scale bar $=10 \mu \mathrm{m}$. 

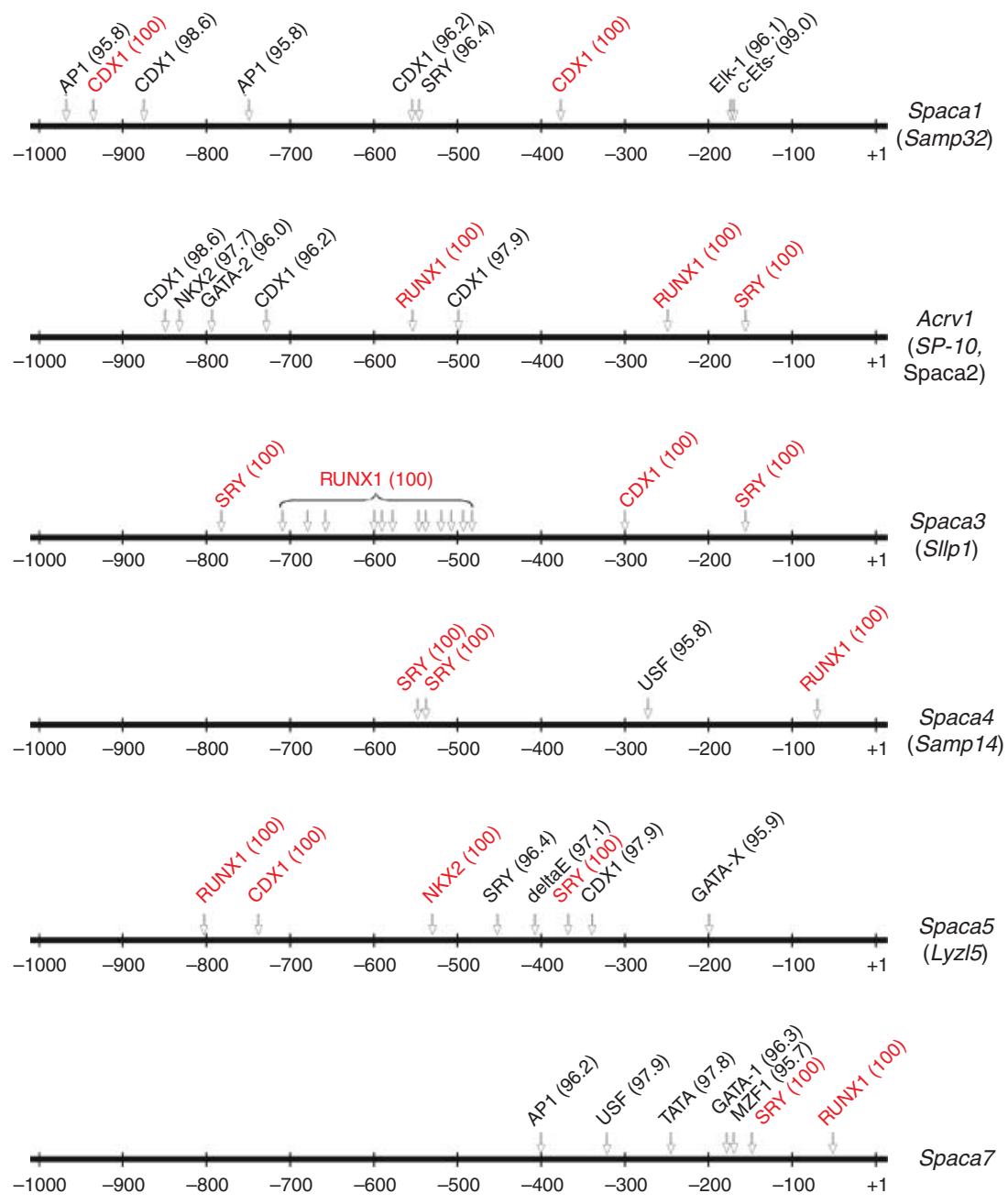

Figure 7 Schematic representation of the putative transcriptional regulatory elements in the $1000 \mathrm{bp}$ promoter of the mouse Spaca family genes (mouse Spaca6 sequence is not provided by NCBI Database). Transcription initiation start site $(+1)$ was provided by the Database of Transcriptional Start Sites (http://dbtss.hgc.jp/; Yamashita et al. 2010). Consensus sequences were sought in the TFSEARCH database (http://mbs.cbrc.jp/research/ db/TFSEARCH.html). Only scores over 95 points are shown (values given in parentheses). Max scores: 100.0 points - shown in red. an another member of the SOX gene family, is restricted to postmeiotic germ cells, at its highest level in round spermatids (Denny et al. 1992), which makes Sox5 a good candidate for spermatid-expressed gene regulator. However, it should be verified experimentally whether members of the SOX family are really involved in the regulation of Spaca gene expression. Binding sites for RUNX1 and caudal type homeobox 1 (CDX1, CdxA) are also common in Spaca promoters, but expression of Runx 1 and $C d x 1$ in adult mouse testes has not been reported.

In summary, SPACA7 is presumed to be a novel acrosomal protein that has no similarity with other members of the SPACA family. Its expression is limited to testes and its precise function is not known at the moment. Further investigation of the physiological roles in reproduction is needed.

\section{Materials and Methods}

\section{Cell culture}

Mouse cell lines originating from embryonic fibroblasts (NIH3T3 and RetroPack PT67) were routinely cultured at $37^{\circ} \mathrm{C}$ and $5 \% \mathrm{CO}_{2}$ in DMEM medium (Sigma) supplemented with $10 \%(\mathrm{v} / \mathrm{v})$ heat-inactivated FBS (ICN Pharmaceuticals, Inc.) and $40 \mu \mathrm{g} / \mathrm{ml}$ gentamicin sulfate (Krka dd Novo Mesto, Novo Mesto, Slovenia). Mouse cell lines originating from lung carcinoma (LL2), endothelial cells of peripheral lymph nodes (HECa10), melanoma (B16-F10), colon adenocarcinoma (C26), and renal carcinoma (Renca) were grown in RPMI (Sigma) supplemented with FBS and gentamicin sulfate as earlier. Cultures were maintained in a humidified $5 \% \mathrm{CO}_{2}$ atmosphere at $37^{\circ} \mathrm{C}$.

\section{RNA isolation, CDNA synthesis, and RT-PCR}

Total RNA was isolated using the GeneMATRIX Universal RNA Purification Kit (Eurix, Gdańsk, Poland). Next, 25-50 $\mu \mathrm{g}$ RNA was incubated with $2.5 \mathrm{U}$ DNase I (Worthington, Lakewood, NY, USA) for $15-30 \mathrm{~min}$ at $37^{\circ} \mathrm{C}$ in a buffer containing $40 \mathrm{mM}$ Tris, $\mathrm{pH} 7.9,10 \mathrm{mM} \mathrm{NaCl}, 6 \mathrm{mM} \mathrm{MgCl}$, and $0.1 \mathrm{mM} \mathrm{CaCl}_{2}$. Reaction was stopped by heating for $5 \mathrm{~min}$ at $75^{\circ} \mathrm{C}$ and RNA was precipitated, washed, dissolved in RNase-free water, and checked for DNA contamination by PCR (35 cycles) with Gapdh primers. For cDNA synthesis, $1 \mu \mathrm{g}$ RNA was primed with random pentadecamer primers (200 ng) (Stangegaard 
et al. 2006) and 18-mer oligo-dT primers (250 ng). Reaction was carried out at $37^{\circ} \mathrm{C}$ for $50 \mathrm{~min}$ in the presence of $5 \mu \mathrm{M}$ dNTPs and $200 \mathrm{U}$ reverse transcriptase (Gibco/Invitrogen) in a total volume of $20 \mu \mathrm{l}$. Reaction was terminated by heating at $70{ }^{\circ} \mathrm{C}$ for $15 \mathrm{~min}$, samples were diluted using water to $400 \mu \mathrm{l}$, aliquoted, and stored at $-20{ }^{\circ} \mathrm{C}$ until use. For RT-PCR, 1-3 $\mu \mathrm{l}$ cDNA template were used and 15-36 cycles were applied depending on the primer set. For each primer set, an optimal annealing temperature was established. Primers used in analyses are listed in Table 1.

\section{Engineering of Spaca7/EGFP fusion constructs}

Sequence coding for putative uncharacterized protein C13orf28 homolog precursor (Spaca7; previously RIKEN cDNA 1700094C09 gene; ID: 78634; GenBank accession no. NM_024279) was amplified by PCR using cDNA from FVB/ $\mathrm{N}$ adult mouse testis as a template. Into forward primer (Table 1), designed upstream of the Spaca7 ATG codon, the sequence recognized by HindIII restriction enzyme was introduced. Into reverse primer, designed around the Spaca7 stop codon, mutation STOP to Ser codon and the sequence recognized by BamHI restriction enzyme were introduced in a position enabling the fusing of Spaca7 coding sequence in a frame with EGFP coding sequence from the pEGFP1 plasmid (GenBank accession no. U55761; Clontech Laboratories, Inc.). A spacer encoding ten amino acids was introduced between the Spaca7 and the EGFP genes' sequences to reduce the possible interference between fused proteins. The PCRamplified Spaca7 fragment was digested with HindIII and BamHI, purified and cloned, together with BamHI-Notl fragment of the pEGFP1 plasmid, between HindIII and Notl in multicloning site of $p L N C X 2$ plasmid (Clontech). In the resulting $p L N C X 2-S p a c a 7 / E G F P$ plasmid, fusion protein was driven by immediate early CMV promoter. For construction of the SPACA7/EGFP fusion protein under control of spermatocyte-specific promoter, Xhol-Notl fragment was recloned from pLNCX2-Spaca7/EGFP into pHST(1195/62)-EGFP+INS plasmid (described in Rupik et al. (2006)). In the resulting plasmid, named here $\mathrm{pH}$ spa2-Spaca7/EGFP, fusion protein was driven by a Kpnl(-1195)-Drall(-62) fragment of the rat Hspa2 promoter (coordinates of the restriction sites refer to the $A(+1)$ in ATG codon of the Hspa2 gene, GenBank accession no. X15705). Additionally, insulator sequences from the chicken $\beta$-globin locus (gift of Dr G Fenselfeld and Dr A West; Bell et al. 1999) flanked the transgene to reduce the chromosomal position effect. The DNA sequence of the recombinant plasmids was confirmed by sequencing.

\section{Transfection of somatic cells}

The pLNCX2-Spaca7/EGFP construct and pLNCX2 as a negative control were introduced into RetroPack PT67 cells (Clontech). The transfections were done using Lipofectamine2000 (Invitrogen) according to the manufacturer's protocol. For each transfection, $8 \mu \mathrm{g}$ DNA was used. To select cells that stably express the integrated vector, cells were cultured with G-418 (Gibco/Invitrogen) at concentration $0.8 \mathrm{mg} / \mathrm{ml}$ for 2 weeks. For monitoring of the fusion protein localization, pLNCX2-Spaca7/EGFP transfected cells and pLNCX2 transfected cells were seeded on Permanox Chamber Slides (Nalge Nunc Int., Rochester, NY, USA).

\section{Generation of transgenic mice}

The pHspa2-Spaca7/EGFP plasmid was digested (EcoRI and Sphl) in order to remove vector sequences and linear DNA was microinjected into the pronuclei of zygotes from $\mathrm{FVB} / \mathrm{N}$ females by standard procedures. Transgenic founders were screened by PCR using genomic DNA isolated from tail biopsies and primers complementary to the Spaca7 and EGFP coding sequences (Table 1). Transgenic mice containing 1133 bp Kpnl-Drall fragment of the rat Hspa2 promoter linked to EGFP gene ( $p H S T(1195 / 62)-E G F P+I N S$ transgene, here designated as $p H s p a 2-E G F P$ transgene) were described in detail elsewhere (Rupik et al. 2006, Vydra et al. 2009). The animals were maintained under controlled environmental conditions with a $12 \mathrm{~h}$ light:12 h darkness cycle and were

Table 1 Characteristics of primers used in analyses.

\begin{tabular}{|c|c|c|c|c|c|}
\hline Gene symbol & $\begin{array}{l}\text { NCBI Ref. } \\
\text { Seq. or Gene ID }\end{array}$ & $\begin{array}{l}\text { Primer sequences } \\
\left(5^{\prime}-3^{\prime}\right)\end{array}$ & $\begin{array}{c}\text { PCR } \\
\text { product } \\
\text { length }(b p)\end{array}$ & $\begin{array}{l}\text { Position in } \\
\text { the ref. } \\
\text { sequence }\end{array}$ & Comments \\
\hline Spaca7 & NM_024279 & $\begin{array}{l}\text { F: CAAAGGAGGAAAGTGGCAAA } \\
\text { R: CTAAGATGGGTGGCATGGAG }\end{array}$ & 312 & $398-709$ & $\begin{array}{l}\text { Used in RT-PCR for endogenous } \\
\text { Spaca7 expression detection } \\
\text { (reverse primer in } 3^{\prime}-U T R \text { ) }\end{array}$ \\
\hline Spaca7 & NM_024279 & $\begin{array}{l}\text { F: TGCAGCCGATCAAGACAACTTC } \\
\text { R: CCAGCATTTATTTCCTTGGTTTGT }\end{array}$ & 196 & $128-323$ & $\begin{array}{l}\text { Used in RT-PCR for Spaca7 and } \\
\text { transgene expression detection }\end{array}$ \\
\hline Spaca7 & NM_024279 & $\begin{array}{l}\text { F: TCCTCCTGAAGCTTGAGGGACCAT } \\
\text { R: CTTGGTCGGATCCTTCTCCTC CGA } \\
\text { AAAGAT }\end{array}$ & 592 & $36-627$ & $\begin{array}{l}\text { Used for cloning. Restriction sites are } \\
\text { underlined; position of mutated } \\
\text { STOP codon is in bold and italic }\end{array}$ \\
\hline $\begin{array}{l}\text { Transgene } \\
\text { Spaca7/EGFP }\end{array}$ & & $\begin{array}{l}\text { F: CAAAGGAGGAAAGTGGCAAA } \\
\text { R: GAACTTCAGGGTCAGCTTGC }\end{array}$ & 377 & & $\begin{array}{l}\text { Used for genotyping and in RT-PCR for } \\
\text { transgene expression detection }\end{array}$ \\
\hline Hspa2 & NM_008301 & $\begin{array}{l}\text { F: AGGGCCCACCATCGAGGAAGTG } \\
\text { R: TCAAGCAAATCTCCATGTACATAC }\end{array}$ & 344 & $2039-2382$ & Used in RT-PCR \\
\hline $18 S$ rRNA & NR_003278 & $\begin{array}{l}\text { F: GTTGGTGGAGCGATTTGTCTGG } \\
\text { R: GCAGCCCCGGATCTAAGG }\end{array}$ & 177 & $1345-1521$ & Used in RT-PCR as reference gene \\
\hline
\end{tabular}

F, forward primer; $R$, reverse primer. 
provided with food and tap water. The animal experiments were approved by the Committee of Ethics and Animal Experimentation.

\section{Monitoring of EGFP expression}

Transfected PT67 cells were monitored for EGFP fluorescence without any fixation. Culture medium was removed from Permanox Chamber Slides (Nunc), cells were gently washed using fresh, $\mathrm{CO}_{2}$-saturated DMEM medium without phenol red, and coverslip was applied (mineral oil was used on the edges to protect against evaporation). For the monitoring of EGFP fluorescence in spermatozoa, caput or cauda of epididymis were dissected in PBS, incubated for 5-10 min in 4\% buffered formaldehyde, and washed in PBS. This step reduced motility of sperm, which allowed to take a picture. Spermatozoa were released directly on a microscopic slide by cutting epididymis in PBS drop. For paraffin sections, testes were fixed in $4 \%$ buffered formaldehyde for $6-8 \mathrm{~h}$ (prolonged fixation reduced green fluorescence) at $4{ }^{\circ} \mathrm{C}$, washed three times for $2-6 \mathrm{~h}$ in PBS at $4{ }^{\circ} \mathrm{C}$, and embedded in paraffin according to Walter et al. (2000), with some modifications. Embedding solvents and times were as follows: $70 \%$ ethanol for $1 \mathrm{~h}$ at room temperature, three times $96 \%$ ethanol for $1 \mathrm{~h}$, three times $100 \%$ ethanol for $20 \mathrm{~min}$, three times xylene for $40 \mathrm{~min}$, and two times paraffin for $1 \mathrm{~h}$ at $58{ }^{\circ} \mathrm{C}$, embedding in paraffin at $58{ }^{\circ} \mathrm{C}$. Sections were cut $6 \mu \mathrm{m}$ thick and mounted on glass slides pretreated with poly-L-lysine. They were deparaffinized in xylene and rehydrated in a descending series of ethanol. When indicated, sections were counterstained for 2-5 min with propidium iodide diluted in PBS to a concentration of $0.2 \mu \mathrm{g} / \mathrm{ml}$ and washed for $15 \mathrm{~min}$ in PBS. Specimens monitored for EGFP fluorescence were mounted in DAKO fluorescence mounting medium. Control, nontransgenic tissues were processed and analyzed in a similar way as transgenic. Observations were made using a microscope (ECLIPSE E800 Nicon). Pictures were recorded with an image analyzer equipped with a Hamamatsu Color Chilled 3 CCD. Additionally, intracellular localization of SPACA7/EGFP fusion protein in spermatozoa was observed under a Carl Zeiss LSM 510 confocal microscope using a $63 \times$ oil immersion objective (NA 1.25). Specimens were illuminated with the $488 \mathrm{~nm}$ excitation line. The emission was collected by a photomultiplier tube after passing through a $505 \mathrm{~nm}$ longpass emission filter. The pinhole was set to $128 \mu \mathrm{m}$. Z-stack images were collected at the format $1024 \times 1024$ pixels. The thickness of each optical slice was $\sim 1 \mu \mathrm{m}$, and the optimal interval between slices was $\sim 0.6 \mu \mathrm{m}$.

\section{Immunolocalization of SPACA7 on human sperm}

A pool of fresh ejaculates was washed in PBS. Suspension of spermatozoa was applied onto microscope slides and air-dried overnight at room temperature. The cells were fixed in $4 \%$ paraformaldehyde for $15 \mathrm{~min}$. Following three washes with PBS for 10 min each, slides were blocked with $2.5 \%$ normal horse serum for $40 \mathrm{~min}$. Sperm were then incubated overnight at $+4{ }^{\circ} \mathrm{C}$ with rabbit polyclonal antibody against human SPACA7 (sc-84020; Santa Cruz Biotechnology, Inc., Santa Cruz, CA, USA) at a concentration of $5 \mu \mathrm{g} / \mathrm{ml}$ in $1 \%$ BSA/PBS.
Negative controls for specificity of the staining were performed by omitting the primary antibody. After washing in PBS $3 \times$ for $5 \mathrm{~min}$ at room temperature, cells were incubated for $40 \mathrm{~min}$ with peroxidase-conjugated ImmPRESS antirabbit Ig reagent (Vector Laboratories, Inc., Burlingame, CA, USA), washed again, and incubated with $0.05 \%$ diaminobenzidine (Vector Laboratories, Inc.) for visualization. The specimens were counterstained with hematoxylin and washed in tap water. Coverslips were then mounted with DAKO Faramount Aqueous Mounting Medium. Slides were observed with a light microscope.

\section{Declaration of interest}

The authors declare that there is no conflict of interest that could be perceived as prejudicing the impartiality of the research reported.

\section{Funding}

This work was supported by the Polish Ministry of Science and Higher Education (grant numbers N N301 002439 and N N401 031837) and European Community from the European Social Fund within the INTERKADRA project UDA - POKL-04.01.0100-014/10-00 (to J Korfanty and A Toma).

\section{References}

Abou-Haila A \& Tulsiani DR 2000 Mammalian sperm acrosome: formation, contents, and function. Archives of Biochemistry and Biophysics $\mathbf{3 7 9}$ 173-182. (doi:10.1006/abbi.2000.1880)

Bell AC, West AG \& Felsenfeld G 1999 The protein CTCF is required for the enhancer blocking activity of vertebrate insulators. Cell 98 387-396. (doi:10.1016/S0092-8674(00)81967-4)

Bellvé AR, Cavicchia JC, Millette CF, O’Brien DA, Bhatnagar YM \& Dym M 1977 Spermatogenic cells of the prepuberal mouse. Isolation and morphological characterization. Journal of Cell Biology 74 68-85. (doi:10.1083/jcb.74.1.68)

Braun RE, Peschon JJ, Behringer RR, Brinster RL \& Palmiter RD 1989 Protamine $3^{\prime}$-untranslated sequences regulate temporal translational control and subcellular localization of growth hormone in spermatids of transgenic mice. Genes and Development 3 793-802. (doi:10.1101/gad. 3.6.793)

Buffone MG, Foster JA \& Gerton GL 2008 The role of the acrosomal matrix in fertilization. International Journal of Developmental Biology $\mathbf{5 2}$ 511-522. (doi:10.1387/ijdb.072532mb)

Denny P, Swift S, Connor F \& Ashworth A 1992 An SRY-related gene expressed during spermatogenesis in the mouse encodes a sequencespecific DNA-binding protein. EMBO Journal 11 3705-3712.

Gennaro R, Simonic T, Negri A, Mottola C, Secchi C, Ronchi S \& Romeo D 1986 C5a fragment of bovine complement. Purification, bioassays, amino-acid sequence and other structural studies. European Journal of Biochemistry 155 77-86. (doi:10.1111/j.1432-1033.1986.tb09460.x)

Hao Z, Wolkowicz MJ, Shetty J, Klotz K, Bolling L, Sen B, Westbrook VA, Coonrod S, Flickinger CJ \& Herr JC 2002 SAMP32, a testis-specific, isoantigenic sperm acrosomal membrane-associated protein. Biology of Reproduction 66 735-744. (doi:10.1095/biolreprod66.3.735)

Heinemeyer T, Wingender E, Reuter I, Hermjakob H, Kel AE, Kel OV, Ignatieva EV, Ananko EA, Podkolodnaya OA, Kolpakov FA et al. 1998 Databases on transcriptional regulation: TRANSFAC, TRRD and COMPEL. Nucleic Acids Research 26 362-367. (doi:10.1093/nar/26.1.362)

Herrero MB, Mandal A, Digilio LC, Coonrod SA, Maier B \& Herr JC 2005 Mouse SLLP1, a sperm lysozyme-like protein involved in sperm-egg binding and fertilization. Developmental Biology 284 126-142. (doi:10. 1016/j.ydbio.2005.05.008) 
Horton P, Park K-J, Obayashi T, Fujita N, Harada H, Adams-Collier CJ \& Nakai K 2007 WoLF PSORT: protein localization predictor. Nucleic Acids Research 35 W585-W587. (doi:10.1093/nar/gkm259)

Krawczyk Z, Mali P \& Parvinen M 1988 Expression of testis-specific hsp70 gene-related RNA in defined stages of rat seminiferous epithelium. Journal of Cell Biology 107 1317-1323. (doi:10.1083/jcb.107.4.1317)

Lee TL, Pang AL, Rennert OM \& Chan WY 2009 Genomic landscape of developing male germ cells. Birth Defects Research. Part C, Embryo Today 87 43-63. (doi:10.1002/bdrc.20147)

Mandal A, Klotz KL, Shetty J, Jayes FL, Wolkowicz MJ, Bolling LC, Coonrod SA, Black MB, Diekman AB, Haystead TA et al. 2003 SLLP1, a unique, intra-acrosomal, non-bacteriolytic, c lysozyme-like protein of human spermatozoa. Biology of Reproduction 68 1525-1537. (doi:10. 1095/biolreprod.102.010108)

Modi D, Shah C, Sachdeva G, Gadkar S, Bhartiya D \& Puri C 2005 Ontogeny and cellular localization of SRY transcripts in the human testes and its detection in spermatozoa. Reproduction 130 603-613. (doi:10. 1530/rep.1.00413)

Moreno RD, Ramalho-Santos J, Sutovsky P, Chan EK \& Schatten G 2000 Vesicular traffic and golgi apparatus dynamics during mammalian spermatogenesis: implications for acrosome architecture. Biology of Reproduction 63 89-98. (doi:10.1095/biolreprod63.1.89)

Primakoff P \& Myles DG 2002 Penetration, adhesion, and fusion in mammalian sperm-egg interaction. Science 296 2183-2185. (doi:10. 1126/science.1072029)

Recillas-Targa F, Pikaart MJ, Burgess-Beusse B, Bell AC, Litt MD, West AG, Gaszner M \& Felsenfeld G 2002 Position-effect protection and enhancer blocking by the chicken beta-globin insulator are separable activities. PNAS 99 6883-6888. (doi:10.1073/pnas.102179399)

Rosa PA, Ogata RT \& Zepf NE 1989 Sequence of the gene for murine complement component C4. Journal of Biological Chemistry 264 16565-16572.

Rupik W, Stawierej A, Stolarczyk I \& Widłak W 2006 Promoter of the heat shock testis-specific Hsp70.2/Hst70 gene is active in nervous system during embryonic development of mice. Anatomica Embryologica 211 631-638. (doi:10.1007/s00429-006-0125-x)

Schultz N, Hamra FK \& Garbers DL 2003 A multitude of genes expressed solely in meiotic or postmeiotic spermatogenic cells offers a myriad of contraceptive targets. PNAS 100 12201-12206. (doi:10.1073/pnas. 1635054100)
Shetty J, Wolkowicz MJ, Digilio LC, Klotz KL, Jayes FL, Diekman AB, Westbrook VA, Farris EM, HaoZ, Coonrod SA et al. 2003 SAMP14, a novel, acrosomal membrane-associated, glycosylphosphatidylinositol-anchored member of the Ly-6/urokinase-type plasminogen activator receptor superfamily with a role in sperm-egg interaction. Journal of Biological Chemistry 278 30506-30515. (doi:10.1074/jbc.M301713200)

Sigrist CJ, Cerutti L, de Castro E, Langendijk-Genevaux PS, Bulliard V, Bairoch A \& Hulo N 2010 PROSITE, a protein domain database for functional characterization and annotation. Nucleic Acids Research 38 D161-D166. (doi:10.1093/nar/gkp885)

Stangegaard M, Dufva IH \& Dufva M 2006 Reverse transcription using random pentadecamer primers increases yield and quality of resulting cDNA. Biotechniques 40 649-657. (doi:10.2144/000112153)

Vydra N, Winiarski B, Rak-Raszewska A, Piglowski W, Mazurek A, Scieglinska D \& Widłak W 2009 The expression pattern of the 70-kDa heat shock protein Hspa2 in mouse tissues. Histochemistry and Cell Biology 132 319-330. (doi:10.1007/s00418-009-0605-1)

Walter I, Fleischmann M, Klein D, Muller M, Salmons B, Gunzburg WH, Renner M \& Gelbmann W 2000 Rapid and sensitive detection of enhanced green fluorescent protein expression in paraffin sections by confocal laser scanning microscopy. Histochemical Journal 32 99-103. (doi:10.1023/A:1004014211408)

Yamashita R, Wakaguri H, Sugano S, Suzuki Y \& Nakai K 2010 DBTSS provides a tissue specific dynamic view of transcription start sites. Nucleic Acids Research 38 D98-D104. (doi:10.1093/nar/gkp1017)

Yan W 2009 Male infertility caused by spermiogenic defects: lessons from gene knockouts. Molecular and Cellular Endocrinology 306 24-32. (doi:10.1016/j.mce.2009.03.003)

Zwingman T, Fujimoto H, Lai LW, Boyer T, Ao A, Stalvey JR, Blecher SR \& Erickson RP 1994 Transcription of circular and noncircular forms of Sry in mouse testes. Molecular Reproduction and Development 37 370-381. (doi:10.1002/mrd.1080370403)

Received 28 July 2011

First decision 5 September 2011

Revised manuscript received 6 February 2012

Accepted 10 April 2012 